\title{
A Protocol for Improved Precision and Increased Confidence in Nanoparticle Tracking Analysis Concentration Measurements between 50 and $120 \mathrm{~nm}$ in Biological Fluids
}

OPEN ACCESS

Edited by:

Rory R. Koenen,

Maastricht University, Netherlands

Reviewed by: Shangfeng Du, University of Birmingham,

United Kingdom

Matthew Dean Linden, University of Western Australia,

Australia

*Correspondence:

Patricia B. Maguire patricia.maguire@ucd.ie

Specialty section:

This article was submitted

to Atherosclerosis and

Vascular Medicine,

a section of the journal

Frontiers in Cardiovascular

Medicine

Received: 18 September 2017

Accepted: 19 October 2017

Published: 03 November 2017

Citation:

Parsons MEM, MCParland D,

Szklanna PB, Guang MHZ,

O'Connell K, O'Connor HD,

McGuigan C, Ní Áinle F, McCann A and Maguire PB (2017) A Protocol for Improved Precision and Increased Confidence in Nanoparticle Tracking Analysis Concentration Measurements between 50 and $120 \mathrm{~nm}$ in Biological Fluids.

Front. Cardiovasc. Med. 4:68. doi: 10.3389/fcvm.2017.00068

\begin{abstract}
Martin E. M. Parsons ${ }^{1,2}$, Damien McParland ${ }^{3}$, Paulina B. Szklanna ${ }^{1,2}$, Matthew Ho Zhi Guang, ${ }^{4,5}$ Karen O'Connell $4,5,6$, Hugh D. O'Connor', Christopher McGuigan ${ }^{4,5,6}$, Fionnuala Ní Áinle ${ }^{1,4,5,8}$, Amanda McCann ${ }^{4,5}$ and Patricia B. Maguire ${ }^{1,2,9 *}$
\end{abstract}

\begin{abstract}
'SPHERE Research Group, UCD Conway Institute, University College Dublin (UCD), Dublin, Ireland, ${ }^{2}$ School of Biomolecular and Biomedical Science, University College Dublin (UCD), Dublin, Ireland, ${ }^{3}$ School of Mathematics and Statistics, University College Dublin (UCD), Dublin, Ireland, ${ }^{4}$ School of Medicine, College of Health and Agricultural Science (CHAS), University College Dublin (UCD), Dublin, Ireland, ${ }^{5}$ UCD Conway Institute of Biomolecular and Biomedical Science, University College Dublin (UCD), Dublin, Ireland, ${ }^{6}$ Department of Neurology, St. Vincent's University Hospital, Dublin, Ireland, ${ }^{7}$ Department of Obstetrics, Rotunda Hospital, Dublin, Ireland, ${ }^{8}$ Department of Haematology, Mater Misericordiae University Hospital, Dublin, Ireland, ' UCD Institute for Discovery, O'Brien Centre for Science, University College Dublin (UCD), Dublin, Ireland
\end{abstract}

Nanoparticle tracking analysis (NTA) can be used to quantitate extracellular vesicles (EVs) in biological samples and is widely considered a useful diagnostic tool to detect disease. However, accurately profiling EVs can be challenging due to their small size and heterogeneity. Here, we aimed to provide a protocol to facilitate high-precision particle quantitation by NTA in plasma, the supernatant of activated purified platelets [the platelet releasate (PR)] and in serum, to increase confidence in NTA particle enumeration. The overall variance and the precision of NTA measurements were quantified by root mean square error and relative standard error. Using a bootstrapping approach, we found that increasing video replicates from $5 s \times 60 s$ to $25 s \times 60 s$ captures led to a reduction in overall variance and a reproducible increase in the precision of NTA particleconcentration quantitation for all three biofluids. We then validated our approach in an extended cohort of 32 healthy donors. Our results indicate that for vesicles sized between 50 and $120 \mathrm{~nm}$, the precision of routine NTA measurements in serum, plasma, and PR can be significantly improved by increasing the number of video replicates captured. Our protocol provides a common platform to statistical compare particle size distribution profiles in the exosomal-vesicle size range across a variety of biofluids and in both healthy donor and patient groups.

Keywords: nanoparticle tracking analysis, extracellular vesicles, plasma, serum, platelets, platelet releasate, particle enumeration

Abbreviations: EVs, extracellular vesicles; FTLA, finite track length adjustment; NTA, nanoparticle tracking analysis; PR, platelet releasate; RMSE, root mean square error; RSE, relative standard error. 


\section{INTRODUCTION}

Extracellular vesicles (EVs) are released by nearly all eukaryotic cells and are found in a diverse range of human biofluids. They regulate a diverse range of biologic and inflammatory pathologic processes and have been implicated in myriad of diseases (1-3). As such, they have emerged as a promising source of future biomarkers in biofluids with both diagnostic and prognostic value $(4,5)$. However, accurately profiling EVs can be challenging due to their small size and heterogeneity (6). While important advances have been made (5), optimization of procedures for $\mathrm{EV}$ quantification across laboratories is of great significance to the EV field with implications for both basic and clinical research.

Nanoparticle tracking analysis (NTA) is widely used to determine the particle size distribution of a sample (6-9). Particle size distribution describes the concentration of particles as a function of size (10). In brief, particle size is determined by focusing a laser beam through a suspension of particles. The light scattered by individual particles in solution allows visualization of particles and each individual particles' displacement is recorded over disjointed time intervals $(8,11,12)$. The mean square displacement for each particle is then used, alongside liquid temperature and viscosity, to calculate individual particle sizes using the Stokes-Einstein equation. The concentration of particles is determined by counting total particles and representing the concentration as a binned histogram (13).

The validity of particle size distributions for a sample depends on accurate sizing of particles as well as precise concentration measurements. Accuracy is generally within $5 \%$ of the expected particle size once correct hardware and software setting have been applied $(7,14,15)$. However, NTA concentration measurements have been shown to have low precision, due to variation in the number of particles detected between video replicate measurements of the same sample (12). For low particle counts, it has been suggested that increasing video replicates could lead to improved concentration measurements (7).

Here, we sought to determine the effect of increasing video replicates on the precision of particle concentration quantitation using NTA in the biofluids of plasma, serum, and the supernatant of activated purified platelets [the platelet releasate (PR)]. Although plasma and serum are widely used in clinical diagnoses $(2,16,17)$, the $\mathrm{PR}$ is an important biofluid to also include as contains a variety of EVs (18-20) and its contents play a fundamental role in hemostasis, wound healing, and the inflammatory response (21-25). In our analysis, we initially used a bootstrapping approach to investigate the precision of particle concentration measurements in all three biofluids. We found that increasing video replicates led to a reproducible increase in the precision of NTA particle concentration quantitation in these biofluids and we validated our findings in an extended clinical cohort.

\section{MATERIALS AND METHODS}

\section{Plasma, PR, and Serum Isolation}

Human plasma, serum, and platelets were obtained from healthy adult volunteers in accordance with approved guidelines from the UCD research, and with ethical approval from St. Vincent's University Hospital and the Rotunda Hospital Research Ethics committees. All subjects gave their informed written consent according to the declaration of Helsinki.

Isolation of platelet-free plasma and PR was as described (19). Briefly, $44 \mathrm{ml}$ of blood was drawn into acid citrate dextrose blood collection tubes (BD, Franklin Lakes, NJ, USA) and the first $4 \mathrm{ml}$ was discarded. Blood was centrifuged at $150 \times g$ for $10 \mathrm{~min}$ at room temperature and platelet-rich plasma was aspirated. Platelets were pelleted from platelet rich plasma by centrifugation at $720 \times g$ for $10 \mathrm{~min}$ at room temperature and platelet poor plasma was aspirated. Platelets were suspended in a modified Tyrode's buffer $(130 \mathrm{mM} \mathrm{NaCl}, 10 \mathrm{mM}$ trisodium citrate, $9 \mathrm{mM} \mathrm{NaHCO}_{3}, 6 \mathrm{mM}$ dextrose, $0.9 \mathrm{mM} \mathrm{MgCl}_{2}, 0.81 \mathrm{mM}$ $\mathrm{KH}_{2} \mathrm{PO}_{4}, 10 \mathrm{mM}$ Tris $\mathrm{pH}$ 7.4). Platelet count was adjusted to $1 \times 10^{9} / \mathrm{ml}$ and platelets were stimulated with $1 \mathrm{U}$ thrombin $/ \mathrm{ml}$ at $37^{\circ} \mathrm{C}$ in a PAP-4 aggregometer under constant stirring. The supernatant from the thrombin activated platelets was centrifuged three times at $10,000 \times g$ for $10 \mathrm{~min}$ at $4^{\circ} \mathrm{C}$ to remove the aggregated platelets and cell debris, leaving the activated platelet supernatant, or PR.

Serum was prepared by drawing $4 \mathrm{ml}$ whole blood into serum blood collection tubes (BD, Franklin Lakes, NJ, USA) and processed according to manufacturer's instructions. In brief, samples were rested upright for 60 min to allow RBCs to clot. The RBC clot was subsequently pelleted by centrifugation at $1,300 \times g$ for $10 \mathrm{~min}$ and serum was aspirated. All plasma, serum, and PR samples were stored at $-80^{\circ} \mathrm{C}$ prior to NTA analysis.

\section{NTA of Biological Samples}

Particle size distribution in PR, plasma, and serum samples was determined by NTA using a NanoSight NS300 system (Malvern Technologies, Malvern, UK) configured with a $488 \mathrm{~nm}$ laser and a high sensitivity scientific CMOS camera. Samples were diluted (PR 1:20-1:50, plasma 1:100-1:2,000, serum 1:500) in particlefree PBS (Gibco, Waltham, MA, USA) to an acceptable concentration, according to the manufacturers recommendations. Samples were analyzed under constant flow conditions (flow rate $=50$ ) at $25^{\circ} \mathrm{C}$ according to Ref. $(7,26)$. For bootstrapped samples, $30 \mathrm{~s} \times 60 \mathrm{~s}$ successive videos were captured with a camera level of 16. Data were analyzed using NTA 3.1.54 software with a

TABLE 1 | Overall variance of nanoparticle tracking analysis measurements shows an exponential decay in variance as $n$ number bootstrapped video replicates increase.

\begin{tabular}{lccc}
\hline & \multicolumn{3}{c}{$\begin{array}{c}\text { Root mean square error (RMSE) represented as \% of } \\
\boldsymbol{n}=\mathbf{5} \text { video replicates }(\mathbf{\pm S D})\end{array}$} \\
\cline { 2 - 4 } $\boldsymbol{n}$ number & Plasma & Platelet releasate & Serum \\
\hline 5 & 100 & 100 & 100 \\
10 & $70.4 \pm 4.27$ & $70.4 \pm 1.88$ & $70.2 \pm 5.33$ \\
15 & $57.6 \pm 3.81$ & $58.0 \pm 3.58$ & $58.0 \pm 4.93$ \\
20 & $50.5 \pm 4.24$ & $49.9 \pm 3.26$ & $51.4 \pm 3.98$ \\
25 & $44.5 \pm 3.32$ & $45.2 \pm 2.28$ & $45.4 \pm 2.82$ \\
\hline
\end{tabular}

Average RMSE $( \pm S D)$ for each plasma, $P R$ and serum is represented as a percentage of RMSE at $5 \mathrm{~min}$. A consistent \% reduction in RMSE observed for each sample with increased $n$ bootstrapped samples. 
detection threshold of 5 . For the validation cohort, $15 \mathrm{~s} \times 60 \mathrm{~s}$ videos were captured with a camera level of 16 and a detection threshold of 10 .

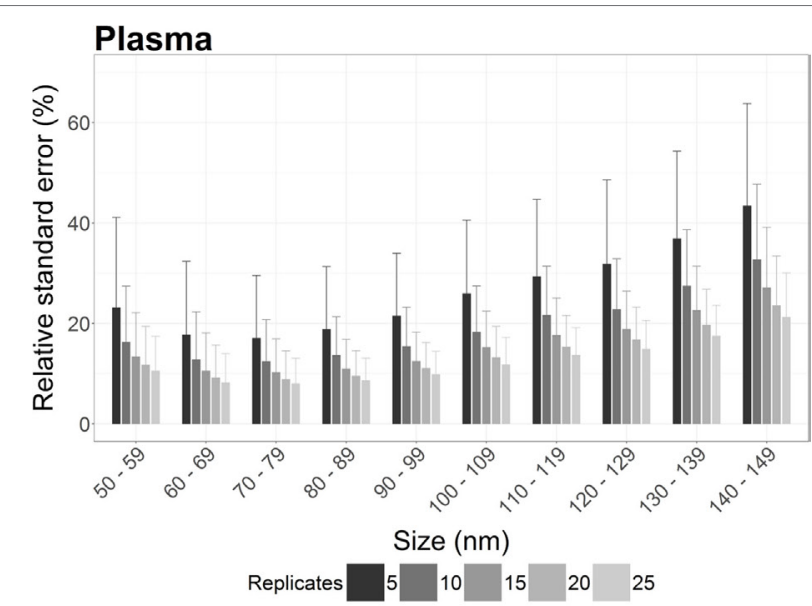

Releasate
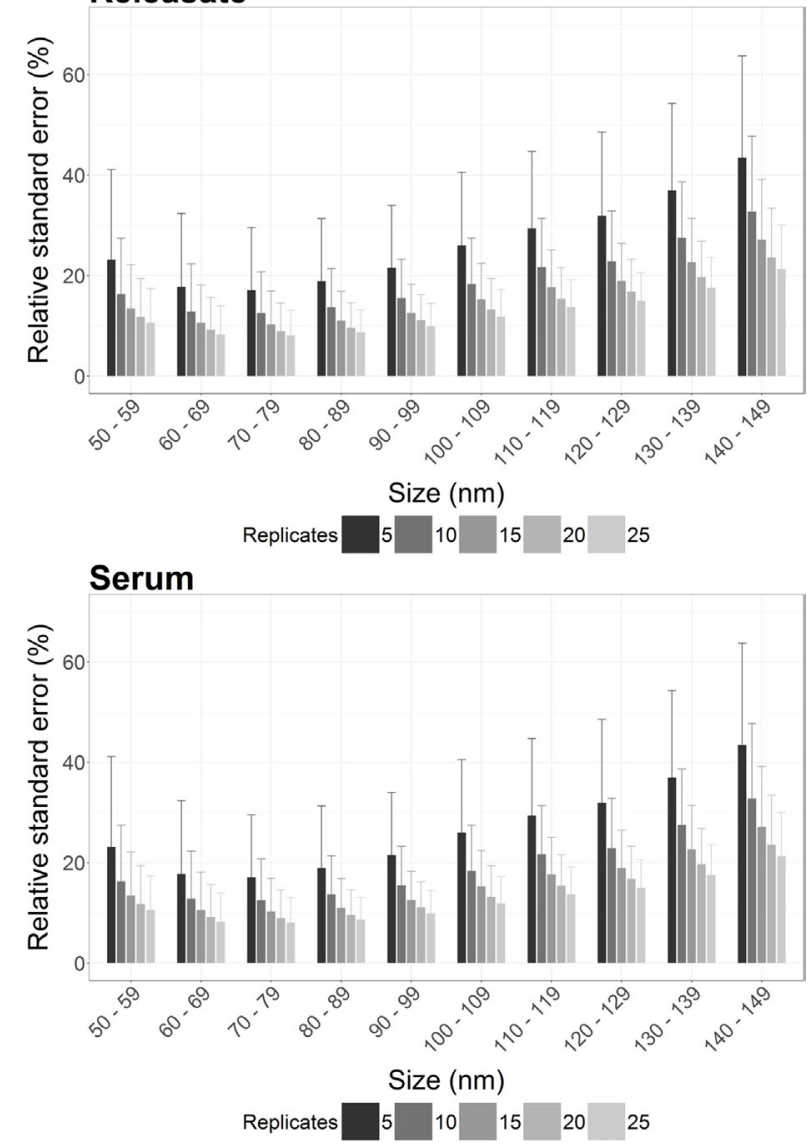

FIGURE 1 | The precision of nanoparticle tracking analysis (NTA) concentration measurements is enhanced by increasing the number of video replicates in all bin widths. For plasma, platelet releasate and serum the average relative standard error (RSE) per bin width from a total of 900 bootstrapped samples was reproducibly decreased by increasing $n$ video replicates. As RSE is a measure of the statistical precision of a sample measurement, the reduction in RSE with increased video replicates indicated that more video replicates led to increased precision of NTA measurements.

\section{Statistical Analysis of Nanoparticle Analysis Tracking Data}

Particle size distributions were binned into $10 \mathrm{~nm}$ bin widths using NTA 3.1.54 software for all video replicates to determine concentration measurements. To understand the variability in the estimates across the complete range of bin widths, we quantified the root mean square error (RMSE) of 100 bootstrap samples of $n=5,10,15,20$, and 25 NTA recordings, which were sampled from the available 30 concentration measurements. The RMSE is the error sum of squares scaled to the data from which it was derived and was calculated as follows:

$$
\operatorname{RSME}_{m}=\sqrt{\frac{\sum_{i=1}^{N_{\text {bins }}} \sum_{j=1}^{N_{\text {boot }}}\left(x_{i j}-\bar{x}_{i}\right)^{2}}{N_{\text {bin }}\left(N_{\text {boot }}-1\right)}}
$$

where $N_{\text {bins }}$ is the number of bins considered, $N_{\text {boot }}$ is the number of bootstrap samples taken for each bin, $x_{\mathrm{ij}}$ is the $j^{\text {th }}$ bootstrap mean for bin $i$ and $\bar{x}_{i}$ is the mean of the bootstrap means for bin $i$.

Next, to determine the precision of the concentration estimates, we quantified the relative standard error (RSE) as follows:

$$
\mathrm{RSE}_{n}=\frac{\frac{s}{\sqrt{n}}}{\bar{c}_{n}} \times 100=\frac{s}{\bar{c}_{n} \sqrt{n}} \times 100
$$

where $s$ is the SD of measurements, $\bar{c}_{n}$ is the sample mean concentration estimate, and $n$ is the number of samples. Precision of our clinical cohort was quantified by RSE where $n=5$ or 15 video replicates. Initially, 100 bootstrap samples were sampled from the available 30 concentration measurements and the RSE was calculated for each bin width for $\mathrm{n}=5,10$, 15,20 , and 25 video replicates. In our validation cohort, RSE was calculated from $n=5$ and $n=15$ video replicates for each bin width.

\section{Statistical Analysis}

All bootstrapping, RMSE, and RSE calculations were performed using the open source programming language for statistical computing R 3.3.1 (27) and the integrated development environment RStudio 1.0.136 (28).

TABLE 2 | The precision of nanoparticle tracking analysis concentration measurement at 100-110 $\mathrm{nm}$ is increased by increasing the number of video replicates.

\begin{tabular}{cccc}
\hline \multirow{2}{*}{$\boldsymbol{n}$ number } & Plasma & Platelet releasate & Serum \\
\cline { 2 - 4 } & $\begin{array}{c}\text { Maximum relative } \\
\text { standard error (RSE) (\%) }\end{array}$ & Maximum RSE (\%) & $\begin{array}{c}\text { Maximum } \\
\text { RSE (\%) }\end{array}$ \\
\hline 5 & 95.3 & & 63.1 \\
10 & 52.8 & 49.2 & 26.6 \\
15 & 41.0 & 25.8 & 20.2 \\
20 & 31.7 & 21.2 & 17.4 \\
25 & 27.6 & 15.5 & 14.2 \\
\hline
\end{tabular}

Focusing on particles from 100 to $110 \mathrm{~nm}$, the maximum RSE is significantly decreased by increasing video replicates. 


\section{RESULTS}

\section{Reduction in the Overall Variance of NTA Measurement by Increasing Video Replicates}

Using a bootstrapping approach, we examined the effect of increasing video replicates on the total variance of NTA measurement of particles in plasma, PR, and serum. RMSE, a measure of total variance, was calculated to show the variability of measurement at each time point. Plasma, PR, and serum were isolated from three healthy donors and subjected to analysis by NTA. $30 \mathrm{~s} \times 60$ s consecutive videos were captured for each sample. All experiments were performed in triplicate. RMSE was calculated for 100 bootstrap samples of $n=5,10,15,20$, and 25 video replicates. RMSE was normalized to $n=5$ video replicates with $n=5$ representing $100 \%$ variance. For plasma, PR, and serum increasing video replicates led to a decrease in total variance across all samples (Table 1). On average, doubling the number of video replicates from $n=5$ to $n=10$ led to a $\sim 30 \%$ reduction in the
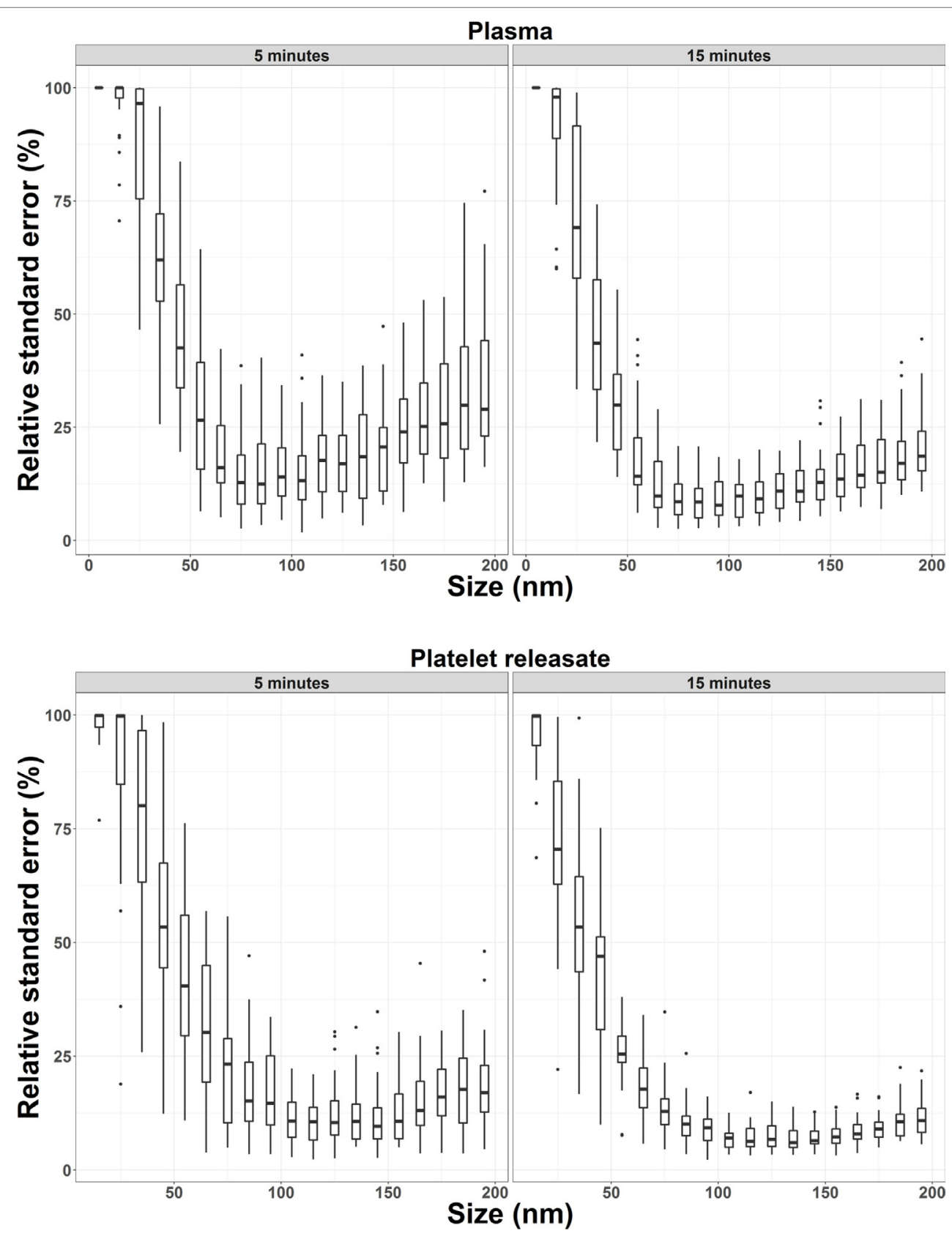

FIGURE 2 | Precision of nanoparticle tracking analysis concentration measurements from 32 healthy donors is increased by increasing the number of video replicates in all bin widths. For plasma and platelet releasate, the average relative standard error per bin width from 32 healthy donors was reproducibly reduced by increasing video replicates from $n=5$ to $n=15$. 
initial variance, with further reductions in total variance reproducibly replicated in all samples with increasing video captures.

\section{Precision of NTA Concentration Measurements in Individual Bin Widths Is Improved with Increasing the Number of Video Captures}

Individual RSE was calculated per $10 \mathrm{~nm}$ bin width for 100 bootstrap samples from plasma, $\mathrm{PR}$, and serum, for $n=5,10,15$, 20 , and 25 video replicates. A reduction in RSE was reproducibly observed across all bin widths for plasma, PR, and serum with increasing video replicates (Figure 1; Table S1 in Supplementary Material).

However, also of interest is the maximum RSE across all bootstrapped samples. Focusing on particles from 100 to $110 \mathrm{~nm}$, the maximum RSE was found to be excessively high for $n=5$ replicates only, with a maximum RSE for plasma of $95.3,49.2 \%$ for PR, and $63.1 \%$ for serum (Table 2). However, increasing $n$ video replicates had a reproducible significant reduction in maximum RSE. For example, for $n=10$ video replicates, maximum RSE for plasma was $52.8,25.8 \%$ for PR, and $26.6 \%$ for serum. Further reductions in maximum RSE were evident with increased $n$ video replicates (Table 2). This pattern was reproduced for all bin widths. The maximal RSE for all bin widths and samples can be found in Table $\mathrm{S} 1$ in Supplementary Material. As RSE is a measure of the precision of concentration measurements by NTA, an overall reduction in maximal RSE indicates that increasing video replicates leads to a reproducible increase in the precision of particle concentration measurements by NTA.

\section{Validation of Increased Precision of NTA Concentration Measurements in a Larger Cohort of 32 Donors}

Next, we wished to determine if the increase in precision of concentration quantitation observed in our bootstrapped samples could be replicated in a larger cohort $(n=32)$. $15 \mathrm{~s} \times 60 \mathrm{~s}$ NTA video replicates were captured for PR and plasma from 32 healthy donors. The first five videos were analyzed to determine the RSE of $n=5$ video replicates for every $10 \mathrm{~nm}$ bin width for each donor. These five, plus an additional 10 videos were then analyzed to determine the RSE of $n=15$ video replicates. By increasing the video replicates from 5 to 15 , the precision of NTA concentration quantitation was improved (Figure 2; Table 3). The maximum RSE in plasma was reduced from 64.37 to $44.34 \%$, while the maximum RSE in PR was reduced from 76.23 to $38.01 \%$. In accordance with the findings from our bootstrapped samples, the precision was most improved in the bin centers corresponding to vesicles of $50-120 \mathrm{~nm}$ in size. For example, taking the bin center from 100 to $110 \mathrm{~nm}$ for illustrative purposes, the maximum RSE was found to be high with $n=5$ replicates with maximum RSE for plasma of $41.0 \%$, and $22.3 \%$ for PR (Table 4). Increasing $n$ video replicates had a reproducible significant effect on maximum RSE. When video replicates were to $n=15$, maximum RSE was 18.0 and $12.6 \%$ for plasma and $\mathrm{PR}$, respectively, indicating a greatly improved precision of NTA concentration measurements (Table 4). In
TABLE 3 | Increased precision of nanoparticle tracking analysis concentration measurements in a larger cohort of 32 healthy donors of particles from 50 to $120 \mathrm{~nm}$.

\begin{tabular}{lcc}
\hline & Plasma & Platelet releasate \\
\cline { 2 - 3 }$n$ & $\begin{array}{c}\text { Maximum relative } \\
\text { standard error (RSE) (\%) }\end{array}$ & Maximum RSE (\%) \\
\hline 5 & 64.37 & 76.23 \\
15 & 44.34 & 38.01 \\
\hline
\end{tabular}

TABLE 4 | Precision of nanoparticle tracking analysis concentration measurements is increased in particles from 100 to $110 \mathrm{~nm}$ by increasing the number of video replicates in a cohort of 32 healthy donors.

\begin{tabular}{|c|c|c|c|c|c|}
\hline \multirow[b]{2}{*}{$n$ number } & \multirow[b]{2}{*}{ Size $(n m)$} & \multicolumn{2}{|c|}{ Plasma } & \multicolumn{2}{|c|}{ Platelet releasate } \\
\hline & & $\begin{array}{l}\text { Average relative } \\
\text { standard error } \\
\text { (RSE) (\%) }\end{array}$ & $\begin{array}{c}\text { Maximum } \\
\text { RSE (\%) }\end{array}$ & $\begin{array}{l}\text { Average } \\
\text { RSE (\%) }\end{array}$ & $\begin{array}{c}\text { Maximum } \\
\text { RSE (\%) }\end{array}$ \\
\hline 5 & $100-110$ & $15.6 \pm 15.6$ & 41.0 & $11.5 \pm 11.5$ & 22.3 \\
\hline 15 & $100-110$ & $9.3 \pm 9.3$ & 18.0 & $6.8 \pm 6.8$ & 12.6 \\
\hline
\end{tabular}

For particles from 100 to $110 \mathrm{~nm}$, average RSE ( $\pm S D)$ is decreased by increasing the number of video replicates. Maximum RSE is also significantly decreased by increasing the number video replicates.

a similar manner, the maximum error was also calculated for each bin width across all samples (Table S2 in Supplementary Material).

\section{DISCUSSION}

Here, we sought to determine the effect of increasing video replicates on the precision of EV concentration quantitation using NTA. Initially, we used a bootstrapping approach to investigate the precision of particle concentration measurements in plasma, the PR, and in serum. Our results indicated that the precision of routine NTA measurements can be significantly improved in the particle size range of 50-120 nm for all biofluids analyzed and this held true even as we extended our analysis to plasma and PR in a larger cohort of 32 donors.

As the NTA method is the current gold standard to measure the size and concentration of small particles in biological samples and has the potential to be a useful diagnostic tool to detect disease $(8,29)$, our protocol has much relevance to the field. In fact, with NTA-acquisition settings constant between analyses, our approach will enable the mean, mode, and median particle size together with EV concentration to be more precisely compared between differential samples. In this way, we conclude that we now provide a common platform to statistically compare particle size distribution profiles from the plasma and PR of patients with a variety of pathologies.

\section{ETHICS STATEMENT}

Human plasma, serum and platelets were obtained from healthy adult volunteers in accordance with approved guidelines from the UCD research ethics committee, and with ethical approval 
from St Vincent's University Hospital and the Rotunda Hospital Research Ethics committees. All subjects gave their informed written consent according to the declaration of Helsinki.

\section{AUTHOR CONTRIBUTIONS}

MP, DM, PS, MG, AM, and PM designed research; MP, DM, PS, MG, KO, HO, CM, FÁ, AM, and PM performed experiments; MP, DM, PS, MG, AM, and PM analyzed data; MP, DM, PS, MG, KO, $\mathrm{HO}, \mathrm{CM}, \mathrm{FÁ}, \mathrm{AM}$, and PM wrote the paper.

\section{FUNDING}

PM is supported by PI award 10/IN.1/B3012 from Science Foundation Ireland. PS is supported by Irish Research Council award GOIPG/2014/575. This research was also funded by a Health Research Board (Ireland) grant (HRA_POR/2013/377). We would like to acknowledge the UCD School of Medicine, University College Dublin, for funding support for the NTA nanoparticle technology equipment (NanoSight NS300, Malvern).

\section{REFERENCES}

1. Yáñez-Mó M, Siljander PR-M, Andreu Z, Bedina Zavec A, Borràs FE, Buzas EI, et al. Biological properties of extracellular vesicles and their physiological functions. J Extracell Vesicles (2015) 4(1):27066. doi:10.3402/jev.v4. 27066

2. Harrison P, Gardiner C, Sargent IL. Extracellular Vesicles in Health and Disease. Boca Raton, FL: CRC Press (2014).

3. van der Pol E, Böing AN, Harrison P, Sturk A, Nieuwland R. Classification, functions, and clinical relevance of extracellular vesicles. Pharmacol Rev (2012) 64(3):676-705. doi:10.1124/pr.112.005983

4. Raposo G, Stoorvogel W. Extracellular vesicles: exosomes, microvesicles, and friends. J Cell Biol (2013) 200(4):373-83. doi:10.1083/jcb.201211138

5. Almutairi MMA, Gong C, Xu YG, Chang Y, Shi H. Factors controlling permeability of the blood-brain barrier. Cell Mol Life Sci (2016) 73(1):57-77. doi:10.1007/s00018-015-2050-8

6. Maas SLN, de Vrij J, van der Vlist EJ, Geragousian B, van Bloois L, Mastrobattista E, et al. Possibilities and limitations of current technologies for quantification of biological extracellular vesicles and synthetic mimics. J Control Release (2015) 200:87-96. doi:10.1016/j.jconrel.2014.12.041

7. Gardiner C, Ferreira YJ, Dragovic RA, Redman CWG, Sargent IL. Extracellular vesicle sizing and enumeration by nanoparticle tracking analysis. $J$ Extracell Vesicles (2013) 2(1).

8. Dragovic RA, Gardiner C, Brooks AS, Tannetta DS, Ferguson DJP, Hole P, et al. Sizing and phenotyping of cellular vesicles using nanoparticle tracking analysis. Nanomedicine (2011) 7(6):780-8. doi:10.1016/j.nano.2011. 04.003

9. Du S, Kendall K, Toloueinia P, Mehrabadi Y, Gupta G, Newton J. Aggregation and adhesion of gold nanoparticles in phosphate buffered saline. J Nanopart Res (2012) 14(3):758. doi:10.1007/s11051-012-0758-z

10. van der Pol E, Coumans FAW, Grootemaat AE, Gardiner C, Sargent IL, Harrison $\mathrm{P}$, et al. Particle size distribution of exosomes and microvesicles determined by transmission electron microscopy, flow cytometry, nanoparticle tracking analysis, and resistive pulse sensing. J Thromb Haemost (2014) 12(7):1182-92. doi:10.1111/jth.12602

11. Saveyn H, De Baets B, Thas O, Hole P, Smith J, Van der Meeren P. Accurate particle size distribution determination by nanoparticle tracking analysis based on 2-D Brownian dynamics simulation. J Colloid Interface Sci (2010) 352(2):593-600. doi:10.1016/j.jcis.2010.09.006

12. Filipe V, Hawe A, Jiskoot W. Critical evaluation of nanoparticle tracking analysis (NTA) by NanoSight for the measurement of nanoparticles and

\section{SUPPLEMENTARY MATERIAL}

The Supplementary Material for this article can be found online at http://www.frontiersin.org/article/10.3389/fcvm.2017.00068/ full\#supplementary-material.

TABLE S1 | Relative standard error per bin width from bootstrap samples. 30 × 60 second consecutive videos were captured for each sample, plasma, platelet releasate (PR), and serum from 3 donors in triplicate. RSE was calculated for 100 bootstrap samples of $n=5,10,15,20$, and 25 video replicates. Average RSE per $\mathrm{n}$ video replicates for each bin size was calculated for all 900 bootstrapped samples. A reduction in RSE was reproducibly observed across all bin widths for plasma, platelet releasate (PR) and serum with increasing video replicates.

TABLE S2 | Relative standard error per bin with from $\mathbf{3 2}$ donor validation. $15 \times 60$ second consecutive videos were captured for each sample, plasma and platelet releasate $(\mathrm{PR})$ for 32 healthy donors. The first five videos were analysed to determine the RSE of $n=5$ video replicates for every $10 \mathrm{~nm}$ bin width for each donor. These five, plus an additional 10 videos were then analysed to determine the RSE of $n=15$ video replicates. The average RSE for each bin size for 5 and 15 minutes shows a reduction for each bin size. The maximum RSE for was reduced for each bin size for both biological samples.

protein aggregates. Pharm Res (2010) 27(5):796-810. doi:10.1007/s11095-010 0073-2

13. Gross J, Sayle S, Karow AR, Bakowsky U, Garidel P. Nanoparticle tracking analysis of particle size and concentration detection in suspensions of polymer and protein samples: influence of experimental and data evaluation parameters. Eur J Pharm Biopharm (2016) 104:30-41. doi:10.1016/j. ejpb.2016.04.013

14. Du S, Kendall K, Morris S, Sweet C. Measuring number-concentrations of nanoparticles and viruses in liquids on-line. J Chem Technol Biotechnol (2010) 85(9):1223-8. doi:10.1002/jctb.2421

15. Röding M, Zagato E, Remaut K, Braeckmans K. Approximate Bayesian computation for estimating number concentrations of monodisperse nanoparticles in suspension by optical microscopy. Phys Rev E (2016) 93(6):063311. doi:10.1103/PhysRevE.93.063311

16. Arraud N, Linares R, Tan S, Gounou C, Pasquet JM, Mornet S, et al. Extracellular vesicles from blood plasma: determination of their morphology, size, phenotype and concentration. J Thromb Haemost (2014) 12(5):614-27. doi:10.1111/jth.12554

17. Anderson NL. The clinical plasma proteome: a survey of clinical assays for proteins in plasma and serum. Clin Chem (2010) 56(2):177-85. doi:10.1373/ clinchem.2009.126706

18. Heijnen HF, Schiel AE, Fijnheer R, Geuze HJ, Sixma JJ. Activated platelets release two types of membrane vesicles: microvesicles by surface shedding and exosomes derived from exocytosis of multivesicular bodies and alpha-granules. Blood (1999) 94(11):3791-9.

19. Coppinger JA, Cagney G, Toomey S, Kislinger T, Belton O, McRedmond JP, et al. Characterization of the proteins released from activated platelets leads to localization of novel platelet proteins in human atherosclerotic lesions. Blood (2004) 103(6):2096-104. doi:10.1182/blood-2003-08-2804

20. Siljander PRM. Platelet-derived microparticles - an updated perspective. Thromb Res (2011) 127(Suppl 2):S30-3. doi:10.1016/S0049-3848(10)70152-3

21. Nurden AT. Platelets, inflammation and tissue regeneration. Thromb Haemost (2011) 105(Suppl 1):S13-33. doi:10.1160/THS10-11-0720

22. Thomas MR, Storey RF. The role of platelets in inflammation. Thromb Haemost (2015) 114(3):449-58. doi:10.1160/TH14-12-1067

23. Martinez CE, Smith PC, Palma Alvarado VA. The influence of platelet-derived products on angiogenesis and tissue repair: a concise update. Front Physiol (2015) 6:290. doi:10.3389/fphys.2015.00290

24. Manne BK, Xiang SC, Rondina MT. Platelet secretion in inflammatory and infectious diseases. Platelets (2017) 28(2):155-64. doi:10.1080/09537104.2016. 1240766 
25. Flaumenhaft R. Platelet secretion. 3rd ed. In: Michelson A, editor. Platelets. London: Academic Press (2012). p. 343-66.

26. Vajen T, Benedikter BJ, Heinzmann ACA, Vasina EM, Henskens Y, Parsons M, et al. Platelet extracellular vesicles induce a pro-inflammatory smooth muscle cell phenotype. J Extracell Vesicles (2017) 6(1):1322454.

27. Team RC. R: A Language and Environment for Statistical Computing. Vienna: R Foundation for Statistical Computing (2016). Available from: https:// www.R-project.org/

28. Team R. RStudio: Integrated Development for R. Boston, MA (2016). Available from: http://www.rstudio.com/

29. Soung YH, Ford S, Zhang V, Chung J. Exosomes in cancer diagnostics. Cancers (2017) 9(1):8. doi:10.3390/cancers 9010008
Conflict of Interest Statement: The authors declare that the research was conducted in the absence of any commercial or financial relationships that could be construed as a potential conflict of interest.

Copyright (® 2017 Parsons, McParland, Szklanna, Guang, O'Connell, O’Connor, McGuigan, Ní Áinle, McCann and Maguire. This is an open-access article distributed under the terms of the Creative Commons Attribution License (CC $B Y)$. The use, distribution or reproduction in other forums is permitted, provided the original author(s) or licensor are credited and that the original publication in this journal is cited, in accordance with accepted academic practice. No use, distribution or reproduction is permitted which does not comply with these terms. 